\title{
Can multiparametric ultrasound improve cognitive MRI/TRUS fusion prostate biopsy
}

\author{
Pietro Pepe, Ludovica Pepe, Paolo Panella, Michele Pennisi \\ Urology Unit, Cannizzaro Hospital, Catania, Italy.
}

\begin{abstract}
Summary Objective: To evaluate the accuracy of multiparametric transrectal ultrasound (contrast-enhanced ultrasound plus elastosonography) in the detection of the suspicious area diagnosed by multiparametric magnetic resonance (mpMRI).

Materials and methods: From June 2018 to June 201960 men (median age 63 years) with persistent suspicion of cancer underwent repeat saturation biopsy following pelvic mpMRI and the lesions characterized by a PI-RADS (Prostate Imaging Reporting and Data System) score $\geq 3$ were submitted to 4 additional cores by transperineal cognitive fusion biopsy (TPBX). All patients, before prostate biopsy, underwent contrast-enhanced ultrasound (CEUS) following intravenous administration of a bolus of Sonovue ${ }^{\circledR}$ (2.4 mg of nonpyrogenic suspension of phospholipid/sulphur hexaphloride); in addition, a transrectal elastosonography (TRES) was done to evaluate prostate tissue elasticity. The accuracy of multiparametric ultrasound to detect the mpMRI lesions was evaluated. Results: In 27/60 (45\%) of men a T1c prostate cancer (PCa) was diagnosed by TPBx and 21 (77.8\%) of them were classified as clinically significant cancer (csPCa); in detail, 16/21 (76.2\%) vs. 5/21 (23.8\%) csPCa were located in the peripheric and anterior zone of the gland, respectively. Median total PSA was $10.3 \mathrm{ng} / \mathrm{ml}$ (range: $4.9-51 \mathrm{ng} / \mathrm{ml}$ ). TRES and CEUS were positive for csPCa only in 6/21 (28.5\%) and 13/21 (62\%) of TPBX showing an increased accuracy directly related with the PI-RADS scores

Conclusions: Multiparametric ultrasound using TRES and CEUS after Sonovue ${ }^{\circledR}$ administration did not improve the accuracy of TPBx in diagnosing csPCa.
\end{abstract}

KEY WORDS: Prostate cancer; Contrast-enhanced ultrasound; Multiparametric ultrasound; Fusion prostate biopsy.

Submitted 11 October 2019; Accepted 23 December 2019

\begin{abstract}
INTRODUCTION
Multiparametric magnetic resonance imaging (mpMRI) combined with transrectal ultrasound (TRUS) fusion targeted biopsy has improved the accuracy of standard biopsy schemes in detecting clinically significant prostate cancer (csPCa) (1-4). A lot of papers refer about the accuracy of mpMRI/TRUS targeted biopsy in the diagnosis of cancer but there are few data about the standardization of the procedure and/or the optimal technique of targeted biopsy (5-7). Although, the in-bore procedure seems to be more accurate to diagnose csPCa in comparion with MRI/TRUS fusion biopsy (61 vs. 47\%) (8) no clinically significant difference has been reported in mul-
\end{abstract}

ticentric clinical trials comparing cognitive vs. fusion vs. in-bore targeted biopsy (5). In the last years, TRUS has been enriched by the introduction of tridimensional and computerized images and by the use of contrast media and transrectal elastosonography (TRES) $(9,10)$, which allow better characterization of intraparenchymal microvasculature. The use of microbubble ultrasound contrast agents (UCA: Sonovue ${ }^{\circledR}$, Definity $^{\circledR}$, Imagent ${ }^{\circledR}$ ) improve flow detection in small vessels distinguish the normal from pathological tissue (11-15).

In addition, the elastosonography measures the degree of distortion of ultrasound beam under the application of an external force that is displayed and scored over the B-mode image in a colour scale that corresponds to tissue elasticity (16-19).

In our series, the accuracy of multiparametric transrectal ultrasound (20) (CEUS plus TRES) in the detection of the suspicious area diagnosed by mpMRI and suitable of targeted transperineal prostate biopsy (TPBx) has been evaluated.

\section{MATERIAL AND METHOdS}

From June 2018 to June 201960 Caucasians men (median age 63 years; range: $47-75$ years) with negative digital rectal examination and previous negative extended biopsy underwent repeat transperineal saturation biopsy (SPBx) for the suspicion of cancer (increasing or persistently elevated PSA values) (21).

After institutional review board and ethical committee approval were granted the informed consent was obtained from all individual participants included in the study. Ten days before SPBx, all the patients underwent pelvic mpMRI. All mpMRI examinations were performed using a 3.0 Tesla scanner, (ACHIEVA 3T; Philips Healthcare Best, the Netherlands) equipped with surface 16 channels phased-array coil placed around the pelvic area with the patient in the supine position; multi-planar turbo spin-echo T2-weighted, axial diffusion weighted imaging, axial dynamic contrast enhanced MRI were performed for each patient. The mpMRI lesions characterized by a PI-RADS (Prostate Imaging Reporting and Data System) version 2 score $\geq 3$ were considered suspicious for cancer. Two radiologists blinded to pre-imaging clinical parameters evaluated the mpMRI data separately and independently.

SPBx (median of 28 cores; range: $26-30$ cores) was per- 
formed transperineally by a Hitachi 70 Arietta ecograph (Chiba, Japan) supplied with a bi-planar transrectal probe (5-7.5 MHz) and using a tru-cut 18 gauge needle (Bard; Covington, GA, USA) under sedation and antibiotic prophylaxis (intravenous administration of 1 gram of Cefazolin before prostate biopsy). One urologist with more than 8 years of experience regarding MRI/TRUS fusion targeted biopsy performed the procedure. In the presence of mpMRI lesions suggestive of cancer a TPBx (four cores) was added to SPBx using the Hitachi 70 Arietta ecograph (10). The data have been collected following the START criteria (22).

All patients, before prostate biopsy, underwent standard TRUS combined with administration of a bolus of Sonovue $^{\circledR}$ (nonpyrogenic suspension of phospholipid/ sulphur hexaphloride) equal to $2.4 \mathrm{mg}$ into a large peripheral vein followed by a flush of saline $(10 \mathrm{ml})$. Before scanning with contrast-enhanced ultrasound (CEUS), an appropriate setup that included low mechanical index (MI) and a split-screen view to display the contrast and B-Mode images at the same time was selected on the Logiq E9 echograph (General Electric; Milwaukee, WI USA) provided of an end-fire transrectal probe.

A timer was activated after UCA injection and the investigation was performed for 200 seconds (median; range 180-240); at the end of the procedure microbubbles were bursted.

Post-contrast imaging began as soon as contrast medium was visible on gray scale continuous harmonic imaging (HI); the microbubbles normally were distributed throughout the prostate, that appeared contrastenhanced, and only areas characterized by the Sonovue ${ }^{\circledR}$ enhancement (15) were considered suspicious for PCa. The TRES evaluation was done before the execution of the targeted cognitive biopsy performing a real-time tissue elastosonography of the gland by the Shear Wave Measurament (SWM) analysis of the prostate (Hitachi 70 Arietta ecograph (Chiba, Japan). After multiparametric ultrasound evaluation the patients were submitted to TPBx plus SPBx (1). The Clavien-Dindo grading system for the classification of biopsy complications was used (23). The detection rate of TPBx in the diagnosis of csPCa (Gleason score $>6$ and/or greatest percentage of cancer $>50 \%$ and/or more than two positive cores) was evaluated (24); in addition, the accuracy of multiparametric ultrasound (elastosonography and/or Sonovue ${ }^{\circledR}$ ) to detect the MRI lesions was evaluated.

\section{RESULTS}

The overall diagnosis of PCa vs. csPCa performing SPBx was equal to $32 / 60(56.7 \%)$ vs. $25 / 60$ (41.7\%) cases, but the data will refer only to the TPBx detection rate for $\mathrm{PCa}$. In 27/60 (45\%) of men a Tlc PCa was

Figure 1. (white arrow).
Table 1.

Clinical and histological parameters in 21 patients with clinically significant prostate cancer (csPCa) diagnosed by cognitive targeted fusion biopsy.

\begin{tabular}{|c|c|}
\hline Biopsy histology and clinical parameters & Number of cases \\
\hline Gleason score $3+4$ (GG2) & 10 cases \\
\hline PIRAD-S score (3 vs. 4 vs. 5 )* & 370 \\
\hline Suspicious TRES & 120 \\
\hline Suspicious CEUS & 240 \\
\hline Gleason score $4+3$ (GG3) & 7 cases \\
\hline PIRAD-S score* & $\begin{array}{lll}3 & 3 & 1\end{array}$ \\
\hline Suspicious TRES & $\begin{array}{lll}1 & 0 & 1\end{array}$ \\
\hline Suspicious CEUS & 121 \\
\hline Gleason score $4+4$ (GG4) & 3 cases \\
\hline PIRAD-S score* & $\begin{array}{lll}0 & 2 & 1\end{array}$ \\
\hline Suspicious TRES & $\begin{array}{lll}0 & 0 & 1\end{array}$ \\
\hline Suspicious CEUS & $\begin{array}{lll}0 & 1 & 1\end{array}$ \\
\hline Gleason score $4+5$ (GG5) & 1 case \\
\hline PIRAD-S score* & $\begin{array}{lll}0 & 0 & 1\end{array}$ \\
\hline Suspicious TRES & $\begin{array}{lll}0 & 0 & 0\end{array}$ \\
\hline Suspicious CEUS & $\begin{array}{lll}0 & 0 & 1\end{array}$ \\
\hline \multicolumn{2}{|c|}{$\begin{array}{l}\text { TRES: transrectal elastosonography; PI-RADS: Prostate Imaging Reporting and Data System; } \\
\text { CEUS: contrast-enhanced ultrasound; GG: Grade Groups ISUP (International Society of Urological } \\
\text { Pathology). }\end{array}$} \\
\hline
\end{tabular}

diagnosed by targeted fusion biopsy and 21 (77.8\%) of them were classified as csPCa (Table 1); in detail, 16/21 (76.2\%) vs. $5 / 21$ (23.8\%) csPCa were located in the peripheric (Figure 1) and anterior zone (Figure 2) of the gland, respectively. Median total PSA was $10.3 \mathrm{ng} / \mathrm{ml}$ (range: $4.9-51 \mathrm{ng} / \mathrm{ml}$ ); the PI-RADS scores, Sonovue ${ }^{\circledR}$ and TRES results are listed in Table 1 . No side-effects were reported after Sonovue ${ }^{\circledR}$ administration; none had significant complications (Clavien-Dindo grade I) from prostate biopsy that needed hospital admission biopsyrelated; moreover, the mpMRI procedure was well tolerated and successfully performed in all cases (men with claustrophobia, cardiac pacemaker and hip replacement were not included in the study).

Sonovue ${ }^{\circledR}$ enhancement in correspondence of the left peripheric zone of the prostate

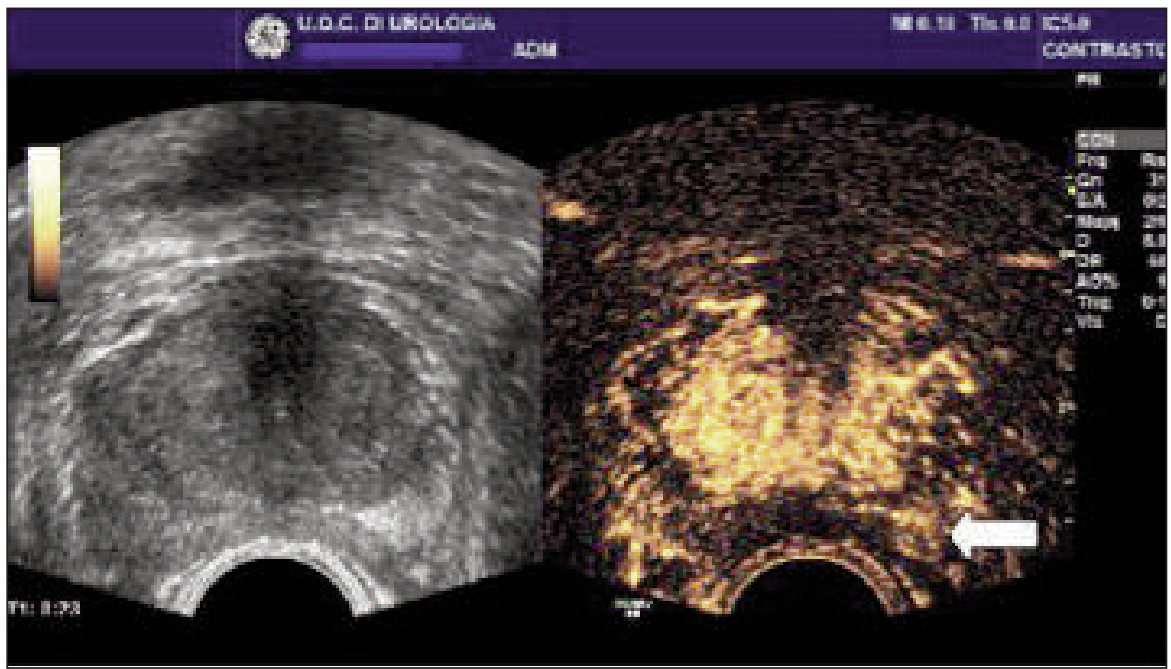


In detail, TRES and CEUS were positive for csPCa only in $6 / 21(28.5 \%)$ and $13 / 21(62 \%)$ of cognitive fusion biopsies showing an increased accuracy directly correlated with the PI-RADS scores (Table 1); in addition, TRES analysis did not improve the CEUS accuracy.

\section{Discussion}

The improvement of diagnostic imaging by mpMRI has allowed targeted biopsies of the suspicious area, increasing the diagnosis of $\operatorname{csPCa}(1,2)$ and reducing the number of unnecessary systematic biopsies. Although mpMRI is strongly recommended in men candidate to prostate biopsy (3), still today, systematic biopsy should be always combined with mpMRI/TRUS fusion biopsy due to false negative rate of mpMRI (about 20\% of the cases) (1) and the variable diagnostic accuracy of the different mpMRI/TRUS fusion biopsy platforms (25). On the other hand, an alternative clinical approach is to begin with mpMRI to determine which patients need a targeted biopsy (26). The detection rate of csPCa is directly related to the PI-RADS score $(1,2)$ and the results depend on clinical parameters, the number of previous negative biopsies and the quality of TPBx procedures. In the next future it would be good that the artificial intelligence for automatic delineation of the prostate on ultrasound could became reliable and applicable to different scanners to improve, guided prostate biopsies using magnetic resonance imaging-transrectal ultrasound fusion (27). Alternatively, the ideal approach to the diagnosis of PCa should be to detect significant disease performing a limited number of targeted biopsy cores improving the accuracy of standard TRUS by multiparametric ultrasound (28); in this respect, a lot of papers have been published on the use of UCA as an additional diagnostic tool for improving PCa diagnosis (2-4) showing a low detection rate included between 15.5 and $32 \%(14-20,29)$. In addition, in recent years, elastosonography has improved by the introduction of Shear Wave Elastosonography (SWE) that is a quantitative method that evaluate local tissue elasticity resulting much less operator dependent; the sensitivity vs. specificity of TRES range from $71-82 \%$ vs. $60-95 \%$ (9) in definitive specimen of men who underwent radical prostatectomy. Recently, Micro-ultrasound (30) in preliminary studies has demonstrated similar sensitivity to clinically significant prostate cancer as mpMRI; unlike mpMRI, micro-ultrasound is performed in the office, in real-time during the biopsy procedure, and so is expected to maintain the cost-effectiveness of conventional ultrasound, but larger studies are needed before these results may be applied in a clinical setting.

In our series, TRES and CEUS were positive for csPCa only in $6 / 21(28.5 \%)$ and $13 / 21(62 \%)$ of cognitive fusion biopsies showing an increased positive results directly related with the PI-RADS scores (Table 1); in addition, TRES analysis did not increased the CEUS accuracy. In definitive, the additional use of multiparametric ultrasound did not improve the accuracy of cognitive fusion biopsy in the diagnosis of csPCa resulting only in an increased cost of the procedure.

Regarding our results some consideration should be done. Firstly, non-targeted biopsies were performed in CEUS or TRES suspicious areas, therefore, it is unknown if multiparametric TRUS would have diagnosed csPCa missed by TPBx; secondly, CEUS was not performed during the execution of TPBx. Third, the false negative rate of mpMRI for csPCa (4/21 equal to $19 \%$ of the cases) has not been correlated to CEUS and TRES findings.

Finally, a greater number of cases is needed to confirm the results.

\section{Conclusions}

Multiparametric ultrasound using TRES plus CEUS did not improved the accuracy of TPBx in diagnosing csPCa.

\section{REFERENCES}

1. Pepe P, Garufi A, Priolo GD, et al. Is it time to perform only MRI targeted biopsy? Ou experience in 1032 men submitted to prostate biopsy. J Urol 2018; 200:774-778.

2. Preisser F, Theissen L, Wenzel M, et al. Performance of combined magnetic resonance imaging/ultrasound fusion-guided and systematic biopsy of the prostate in biopsy-naive patients and patients with prior biopsies. Eur Urol Focus. 2019; pii: S2405-4569(19)30170-1.

3. Pepe P, Garufi A, Priolo G, et al. Can MRI/TRUS fusion targeted biopsy replace saturation prostate biopsy in the re-evaluation of men in active surveillance? World J Urol. 2016; 34:1249-1453.

4. Pepe P, Garufi A, Priolo G, et al. Transperineal versus transrectal MRI/TRUS fusion targeted biopsy: detection rate of clinically significant prostate cancer. Clin Genitourin Cancer. 2017; 15:e33-e36.

5. Wegelin O, Exterkate L, van der Leest $M$, et al. The FUTURE Trial: A Multicenter randomised controlled trial on target biopsy techniques based on magnetic resonance imaging in the diagnosis of prostate cancer in patients with prior negative biopsies. Eur Urol. 2019; 75: 582-590.

6. Galosi AB, Maselli G, Sbrollini $G$, et al. Cognitive zonal fusion biopsy of the prostate: Original technique between target and saturation: Arch Ital Urol Androl. 2016; 88:292-295.

7. Pepe P, Pennisi M, Fraggetta F. How many cores should be obtained during saturation biopsy in the era of multiparametric magnetic resonance? Experience in 875 patients submitted to repeat prostate biopsy. Urology. 2019; pii: S0090-4295(19)31022-2.

8. Costa DN, Goldberg K, Leon AD, et al. Magnetic resonance imaging-guided In-bore and magnetic resonance imaging-transrectal ultrasound fusion targeted prostate biopsies:an adjusted comparison of clinically significant prostate cancer detection rate. Eur Urol Oncol. 2019; 2:397-404.

9. Aboumarzouk OM, Ogston S, Huang Z, et al. Diagnostic accuracy of transrectal elastosonography (TRES) imaging for the diagnosis of prostate cancer: a systematic review and meta-analysis. BJU Int. 2012; 110:1414-1423.

10. Correas JM, Drakonakis E, Isidori AM, et al. Update on ultrasound elastography: miscellanea. Prostate, testicle, musculo-skeletal. Eur J Radiol. 2013; 82:1904-1912.

11. Mitterberger M, Horninger W, Pelzer A, et al: A prospective randomized trial comparing contrast-enhanced targeted versus systematic ultrasound guided biopsies: impact on prostate cancer detection. Prostate. 2007; 67:1537-1542.

12. Colleselli D, Bektic J, Schaefer G, et al. The influence of prostate volume on prostate cancer detection using a combined approach of 
contrast-enhanced ultrasonography-targeted and systematic grayscale biopsy. BJU Int. 2007; 100:1264.

13. Linden RA, Trabulsi EJ, Forsberg F, et al. Contrast enhanced ultrasound flash replenishment method for directed prostate biopsies. J Urol. 2007; 178:2354-2358.

14. Taymoorian K, Thomas A, Slowinski T, et al. Transrectal broadband-Doppler sonography with intravenous contrast medium administration for prostate imaging and biopsy in men with elevated PSA value and previous negative biopsies. Anticancer Res. 2007; 27:4315-4320.

15. Pepe P, Candiano G, Pennisi M, Aragona F. Can Sonovue targeted biopsy replace extended or saturation biopsy in prostate cancer diagnosis? Our experience at primary and repeat biopsy. Arch Ital Urol Androl. 2010; 82:155-159.

16. Nelson ED, Slotoroff CB, Gomella LG, Halpern EJ. Targeted biopsy of the prostate: the impact of color Doppler imaging and elastography on prostate cancer detection and Gleason score. Urology. 2007: 70:1136-1140.

17. Bercoff J, Tanter M, Fink M. Supersonic shear imaging: a new technique for soft tissue elasticity mapping. IEEE Trans Ultrason Ferroelectr Freq Control. 2004; 51:396-409.

18. Nguyen TM, Couade M, Bercoff J, Tanter M: Assessment of viscous and elastic properties of sub-wavelength layered soft tissues using shear wave spectroscopy: theoretical framework and in vitro experimental validation. IEEE Trans Ultrason Ferroelectr Frea Control. 2011; 58:2305-2315.

19. Tanter M, Bercoff J, Athanasiou A, et al. Quantitative assessment of breast lesion viscoelasticity: initial clinical results using supersonic shear imaging. Ultrasound Med Biol. 2008; 34:1373-1386.

20. Mannaerts CK, Wildeboer RR, Remmers S, et al. Multiparametric ultrasound for prostate cancer detection and localization: Correlation of B-mode, shearwave elastography and contrast-enhanced ultrasound with radical prostatectomy specimens. J Urol. 2019 202:11661173.

21. Pepe P, Aragona F. Saturation prostate needle biopsy and prostate cancer detection at initial and repeat evaluation. Urology. 2007; 70:1131-1135

22. Moore CM1, Kasivisvanathan V, Eggener S, et al. START Consortium: Standards of reporting for MRI-targeted biopsy studies (START) of the prostate: recommendations from an International Working Group. Eur Urol. 2013; 64:544-552.

23. Dindo D, Clavien PA. Classification of surgical complications. A new proposal with evaluation in a Cohort of 6336 patients and results of survey. Annals of Surgery. 2004; 2:205-213.

24. Epstein J, Walsh P, Carmichael M. Pathological and clinical findings to predict tumor extent of non palpable (stage T1c) prostate cancer. JAMA. 1994; 271:368-374.

25. Kaufmann S, Russo GI, Bamberg F, et al. Prostate cancer detection in patients with prior negative biopsy undergoing cognitive, robotic or in-bore MRI target biopsy. World J Urol. 2018; 36:761-768.

26. Faria R, Soares MO, Spackman E, et al. optimising the diagnosis of prostate cancer in the era of multiparametric magnetic resonance imaging: a cost-effectiveness analysis based on the prostate MR imaging study (PROMIS). Eur Urol. 2018; 73:23-30.

27. van Sloun RJG, Wildeboer RR, Mannaerts CK, et al. Deep Learning for Real-time, Automatic, and Scanner-adapted Prostate (Zone) Segmentation of Transrectal Ultrasound, for Example, Magnetic Resonance Imaging-transrectal Ultrasound Fusion Prostate Biopsy. Eur Urol Focus. 2019; pii: S2405-4569(19)30125-7.

28. Grey A, Ahmed HU. Multiparametric ultrasound in the diagnosis of prostate cancer. Curr Opin Urol. 2016; 26:114-119.

29. Delgado Oliva F, Arlandis Guzman S, et al. Diagnostic performance of power doppler and ultrasound contrast agents in early imaging-based diagnosis of organ-confined prostate cancer: Is it possible to spare cores with contrast-guided biopsy? Eur J Radiol. 2016; 85:1778-1785

30. Eure G, Fanney D, Lin J, et al. Comparison of conventional transrectal ultrasound, magnetic resonance imaging, and microultrasound for visualizing prostate cancer in an active surveillance population: A feasibility study. Can Urol Assoc J. 2019; 13:E70-E77.

\section{Correspondence}

Pietro Pepe, MD (Corresponding Author)

piepepe@hotmail.com

Ludovica Pepe, MD

Paolo Panella, MD

Michele Pennisi, MD

Divisione di Urologia, Azienda Ospedaliera Cannizzaro,

Via Messina, 829 - 95126 Catania (Italy) 